
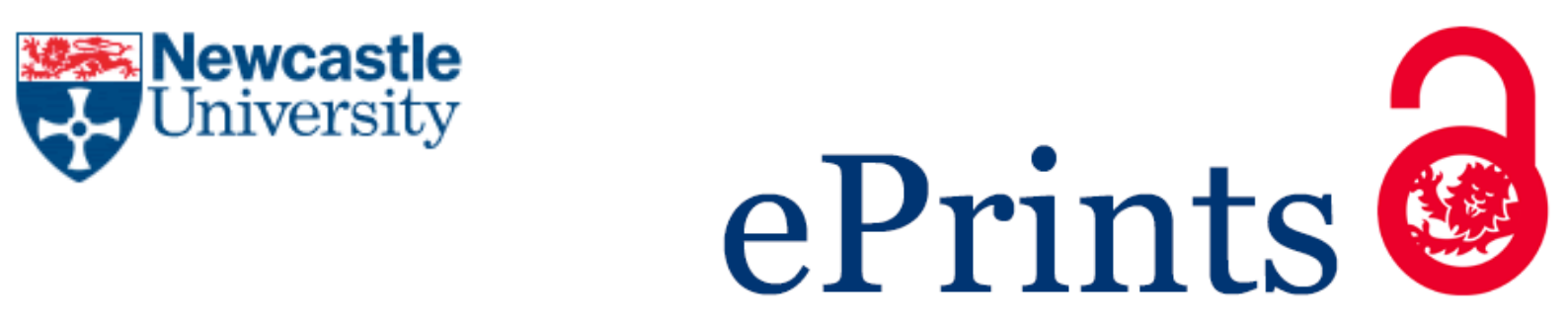

Cauldwell M, Steer PJ, Bonner S, Asghar O, Swan L, Hodson K, Head CEG, Jakes AD, Walker N, Simpson M, Bolger AP, Siddiqui F, English KM, Maudlin L, Abraham D, Sands AJ, Mohan AR, Curtis SL, Coats L, Johnson MR.

Retrospective UK multicentre study of the pregnancy outcomes of women with a Fontan repair. Heart 2017

DOI: $\underline{\text { https://doi.org/10.1136/heartinl-2017-311763 }}$

\title{
Copyright:
}

(C) Article author(s) (or their employer(s) unless otherwise stated in the text of the article) 2017. All rights reserved. No commercial use is permitted unless otherwise expressly granted.

DOI link to article:

https://doi.org/10.1136/heartjnl-2017-311763

Date deposited:

$25 / 01 / 2018$ 


\section{A retrospective UK multi-centre study of the pregnancy outcomes of women with}

a Fontan repair.

Matthew Cauldwell ${ }^{1}$, Philip J Steer ${ }^{1}$ Samantha Bonner ${ }^{2}$, Omar Ashgar ${ }^{3}$, Lorna Swan ${ }^{4}$, Kenneth Hodson ${ }^{5}$, Catherine Head ${ }^{6}$, Adam D Jakes ${ }^{7}$, Niki Walker ${ }^{8}$ Margaret Simpson ${ }^{8}$ Aidan Bolger ${ }^{9}$, Farah Siddiqui, ${ }^{10}$, Katherine M English ${ }^{11}$, Lucy Maudlin ${ }^{12}$, Dilip Abraham ${ }^{13}$, Andrew Sands ${ }^{14}$, Aarthi R Mohan ${ }^{15}$, Stephanie Curtis ${ }^{16}$, Louise Coats ${ }^{17}$, Mark R Johnson ${ }^{1}$

1. Academic Department of Obstetrics and Gynaecology, Chelsea and Westminster Hospital, 369 Fulham Road London, UK, SW10 9NH, mark.johnson@imperial.ac.uk

2. Department of Obstetrics, St Mary's Hospital, Manchester, United Kingdom

3. Manchester Heart Centre, St Mary's Hospital, Manchester, United Kingdom

4. Department of Adult Congenital Heart Disease, Royal Brompton Hospital, London, SW3 6NP

5. Department of Obstetrics, Royal Victoria Infirmary, The Newcastle upon Tyne Hospitals NHS Foundation Trust, Newcastle upon Tyne, United Kingdom

6. Adult Congenital Heart Disease Service, St Thomas' Hospital, London, United Kingdom

7. Department of Obstetrics, St Thomas' Hospital, London, United Kingdom

8. Scottish Adult Congenital Cardiac Service, Golden Jubilee National Hospital, Glasgow, Scotland ,United Kingdom

9. Department of Adult Congenital Heart Disease, Leicester University Hospital, United Kingdom

10. Department of Obstetrics, Leicester University Hospital NHS Trust, United Kingdom

11. Department of Adult Congenital Heart Disease, Leeds Teaching Hospitals NHS Trust, United Kingdom

12. Department of Obstetrics Norfolk and Norwich University Hospital, United Kingdom

13. Department of Adult Congenital Heart Disease, Norfolk and Norwich University Hospital United Kingdom

14. Royal Belfast Hospital for Sick Children, Department of Adult Congenital Heart Disease, Belfast, United Kingdom

15. Department of Obstetrics, University Hospitals Bristol NHS Foundation Trust, Bristol, United Kingdom

16. Department of Adult Congenital Heart Disease, University Hospitals Bristol NHS Foundation Trust Bristol, United Kingdom

17. Department of Adult Congenital Heart Disease, Freeman Hospital, The Newcastle upon Tyne Hospitals NHS Foundation Trust, Newcastle upon Tyne, United Kingdom and Institute of Genetic Medicine, Newcastle University, Newcastle upon Tyne, United Kingdom

Keywords: Fontan procedure, pregnancy, perinatal mortality

\section{Conflict of Interest: None}

Wordcount: 3000 


\begin{abstract}
Background

The population of women of child-bearing age palliated with a Fontan repair is increasing. The aim of this study was to describe the progress of pregnancy and its outcome in a cohort of patients with a Fontan circulation in the United Kingdom (UK).
\end{abstract}

\title{
Methods
}

A retrospective study of women with a Fontan circulation delivering between January 2005 and November 2016 in ten specialist adult congenital heart disease centres in the United Kingdom.

\section{Results:}

50 women had 124 pregnancies, resulting in 68 (54.8\%) miscarriages, two terminations of pregnancy, one intrauterine death (at 30 weeks), 53 (42.7\%) live births and four neonatal deaths. Cardiac complications in pregnancies with a live birth included heart failure $(n=7,13.5 \%)$, arrhythmia $(n=6,11.3 \%)$ and pulmonary embolism $(n=1,1.9 \%)$. Very low baseline maternal oxygen saturations at first obstetric review were associated with miscarriage. All eight women with saturations of less than $85 \%$ miscarried, compared to 60 of 116 $(51.7 \%)$ who had baseline saturations of $\geq 85 \%(p=0.008)$. Obstetric and neonatal complications were common: preterm delivery $(n=39,72.2 \%)$, small for gestational age $\left(<10^{\text {th }}\right.$ percentile, $n=30,55.6 \%,<5^{\text {th }}$ centile $\left.n=19,35.2 \%\right)$ and postpartum haemorrhage $(n=23,42.6 \%)$. There were no maternal deaths in the study period.

\section{Conclusion:}

Women with a Fontan circulation have a high rate of miscarriage and, even if pregnancy progresses to a viable gestational age, a high rate of obstetric and neonatal complications. 


\section{Key Questions}

\section{What is already known about this subject}

Pregnancies in women with a Fontan repair are relatively uncommon and characterized by high rates of cardiac, obstetric and neonatal complications.

\section{What does this study add?}

This is the largest multi-centre study to date of pregnancy outcomes in women with a Fontan repair. We show that maternal cardiac complications are relatively uncommon in contrast to neonatal and obstetric complications, which occur in the majority of pregnancies and most frequently in those with lower baseline maternal oxygen saturations. No association was found between anticoagulation strategy and obstetric outcomes, specifically, miscarriage and post-partum blood loss.

\section{How might this impact upon clinical practice?}

This study will provide clinicians with detailed and contemporary information upon which they can base pre-conception advice for women with a Fontan repair. Furthermore, our study underlines the importance of managing these women in specialist centres, where they will receive appropriate care from the multidisciplinary team. 


\section{Introduction}

The Fontan circulation was devised in 1971 to separate pulmonary and systemic circulations in patients with complex congenital conditions characterized by the presence of a single functional ventricle. Its success in altering the natural history of these conditions means that the already substantial number of women with this condition in the United Kingdom (UK) adult population is expected to increase by around $60 \%$ over the next decade (1); many of them will wish to embark upon a pregnancy. It is clear that women with a Fontan circulation find it difficult to support a pregnancy, probably because the systemic ventricle's ability to increase its cardiac output is compromised by restricted preload and chronically elevated systemic venous pressures (2). Pregnancy also predisposes to the development of atrial arrhythmias $(3,4)$. Prophylactic anticoagulation during pregnancy is advocated by some, but may exacerbate any bleeding tendency and increases the risk of postpartum haemorrhage $(4,5)$. Those with fenestrated circuits or collateral vessels may have cyanosis, which is associated with increased rates of miscarriage and fetal complications including preterm delivery and fetal growth restriction $(6,7)$.

Given these complications, women need comprehensive preconception counseling (PCC) from appropriately experienced multidisciplinary teams (MDT). However there are limited data to inform these discussions (4). The aim of this study was to define pregnancy outcomes from a UK cohort of patients with single ventricle physiology palliated with Fontan repair.

\section{Methods}

\section{Study Population}

All UK specialist centres providing a joint obstetric and adult congenital heart disease (CHD) service were emailed in September 2016 and invited to contribute data on women with a Fontan circulation who had been pregnant. Pseudoanonymized data was collected through review of medical and obstetric case notes. The study period was January 2005 to November 2016. 


\section{Data Collection}

Demographic and baseline maternal data included underlying cardiac anatomy, surgical repair, presence of fenestration, age at Fontan completion, history of arrhythmia or thrombo-embolic events, New York Heart Association class (NYHA), oxygen saturations, anticoagulation regime and qualitative echocardiographic assessment of ventricular function and atrioventricular valve regurgitation. Cardiac Disease in Pregnancy Risk scores (CARPREG) were calculated for all women (8).

Complications were classified as maternal cardiac, obstetric or neonatal. Maternal cardiac complications included arrhythmia, heart failure requiring treatment, systemic thromboembolism, development of new cyanosis (resting maternal saturations <92\%) and protein losing enteropathy. Obstetric complications included pregnancy induced hypertension $(\mathrm{PIH},>140 \mathrm{mmHg}$ systolic or $>90 \mathrm{mmHg}$ diastolic after 20 weeks gestation); pre-eclampsia (PIH with proteinuria $>0.3 \mathrm{~g}$ per 24 hour urine collection), HELLP syndrome (haemolysis, elevated liver enzymes and low platelets); preterm delivery (delivery <37 weeks gestation); postpartum haemorrhage (PPH) (blood loss $\geq 500 \mathrm{ml}$ at vaginal delivery and $\geq 1000 \mathrm{mls}$ at caesarean section). Neonatal complications included preterm birth $(<37$ weeks gestation), small for gestational age (corrected fetal birthweight $<10^{\text {th }}$ centile and $<5^{\text {th }}$ centile $)(9,10)$; stillbirth (fetal demise $\geq 24$ weeks gestation); neonatal death (death within first month of birth); occurrence of congenital heart disease.

\section{Statistics}

Data were analysed using SPSS V.23 for Windows. Continuous variables were expressed as medians, range and interquartile range (IQR). Correlations were calculated using Pearson's product moment if variables were continuous and Spearman rank-order correlation if either of the variables was ordinal.

Differences between continuous variables was assessed with the Mann-Whitney $\mathrm{U}$ test as they were not normally distributed. Categorical data was expressed as absolute numbers and percentages of the denominator and compared using 
Fisher's exact test or the $\chi^{2}$ test if cell numbers were too large. All tests were two-tailed and $\mathrm{p}<0.05$ was considered statistically significant.

\section{Ethics}

Collection of identifiable data previously recorded as part of routine clinical care was restricted to members of the clinical team caring for the women. Metaanalysis was limited to data provided without patient identifiers (pseudoanonymisation) and therefore this study did not require ethical approval (UK National Research Ethics Committee recommendation).

\section{Results}

\section{Study Population}

Of fourteen centres contacted, twelve agreed to participate but two subsequently withdrew due to inability to retrieve necessary clinical information. 50 women had 124 pregnancies. 17 women (34\%) had one pregnancy, 11 (22\%) had two, $15(30 \%)$ had three, two (4\%) had four, three (6\%) had five, one (2\%) had eight and one (2\%) had nine. Thirteen (26\%) women had no viable pregnancies, 26 (52\%) had one, seven (14\%) had two, three (6\%) had three and one $(2 \%)$ had four. There were 53 (42.7\%) livebirths in 37 women. In addition, there were 68 (54.8\%) miscarriages (63 (92.6\%) of these were in the first trimester and five (7.3\%) second trimester), two terminations, one intrauterine death at 30 weeks gestation and four (3.2\%) neonatal deaths. Median age at first pregnancy was 27 years (range 19-36 years, interquartile range (IQR) 24 to 29 years). Underlying congenital heart lesions and type of Fontan repair are shown in Table 1. Median age at Fontan repair was 7 years (range 1-26 years, IQR 4-12 years). Maternal baseline characteristics are shown in Table 2. 42 women (84\%) were white European, eight (16\%) were South Asian. Median body mass index at first pregnancy was 25 (range 18-39, IQR 23-28). Two women smoked pre-pregnancy.

\section{Preconception counseling}

$96(77.4 \%)$ of all pregnancies were planned and 41 (82\%) of women had received PPC prior to their first pregnancy. Of the 12 women whose first 
pregnancy was unplanned, only four (33\%) women had had PCC. Both women who chose a first trimester termination had had PCC

\section{Medication}

\section{Prior to pregnancy}

Table 3 shows cardiac medications prescribed prior to pregnancy in all 53 pregnancies resulting in livebirths.

\section{Anticoagulation and antiplatelet use during pregnancy.}

Anticoagulant and antiplatelet regimens were individualized and involved multidisciplinary decision. In $86(70.5 \%)$ of the 122 pregnancies for which we have medication data, women were taking warfarin pre-pregnancy and one, continued to take warfarin until the third trimester (late presentation). All others stopped warfarin on becoming pregnant. In the 52 of 53 cases where women had a livebirth and anticoagulation details were available, one (1.9\%) took no medication (booked late at 29 weeks), two (3.8\%) took aspirin alone, two (3.8\%) took a prophylactic dose of low molecular weight heparin (LMWH) (clexane $40 \mathrm{mg}$, dalteparin 5000IU or tinzaparin 4500IU - based on booking weight (11)), $12(23.1 \%)$ took prophylactic LMWH and aspirin, 24 (46.2\%) took treatment dose LMWH (clexane twice daily or dalteparin twice daily, based on booking weight, with poor compliance in two cases) and $11(21.2 \%)$ took treatment dose LMWH and aspirin. Women taking treatment dose LMWH had Factor Xa levels performed in accordance with their local guidance. One patient was converted back to warfarin following development of a subchorionic haematoma and recurrent vaginal bleeding felt to be exacerbated by treatment dose LMWH; she ruptured her membranes at 24 weeks and delivered at 26 week with suspected chorioamnionitis.

\section{Infertility}

Three women, underwent in vitro fertilization for unexplained infertility (all had a prior miscarriage). None conceived following in vitro fertilization; one went on to have a successful pregnancy from a spontaneous conception. 


\section{Miscarriage}

68 (54.8\%) of pregnancies ended in miscarriage. Maternal baseline saturations at the first obstetric visit (typically 8-12 weeks) were associated with miscarriage; all eight women with baseline saturations less than $85 \%$ miscarried, compared to 60 of 116 women (51.7\%) with saturations greater than or equal to 85\% (Fisher's exact, $\mathrm{p}=0.008$ ). No significant correlation with miscarriage frequency was seen at saturations greater than or equal to $85 \%$ ( $p=0.595$, Spearman's rank correlation test). The five second trimester ( $\geq 14$ weeks) miscarriages were all associated with recurrent early pregnancy bleeding; subsequently developing a subchorionic haematoma. All women took LMWH (3 prophylactic dose and two treatment dose). It is notable that the median initial haemoglobin concentration in pregnancies ending in miscarriage was 144g/l (range 99-196g/dl, IQR 130-160g/l) whereas in pregnancies with a live birth it was 138g/l (range 109-161g/dl, IQR 130-150g/dl)(Spearman correlation, $\mathrm{p}=0.05$ ). This is substantially higher than in normal pregnancies (mean value 120 SD 7.0 at 12 weeks of pregnancy), the high values likely being a consequence of maternal cyanosis (12). We did not find any correlation between oxygen saturation or haemoglobin concentration and birth weight centile (Pearson's correlation coefficients 0.030 and 0.059 , p values 0.831 and 0.672 respectively). In women with a documented fenestration prior to pregnancy there were 13 potentially viable births from 22 pregnancies (59\%) compared with 41 of 102 $(40.2 \%)$ women without fenestrations $(\mathrm{p}=0.145)$.

\section{Cardiovascular complications in pregnancies progressing beyond 24 weeks} Analyzing both the CARPREG and NYHA score at the beginning of each pregnancy, neither was useful in predicting miscarriage, or birthweight and gestational age in pregnancies with a successful outcome (Spearman correlation, $\mathrm{p}=0.453$ and $0.536 ; \mathrm{p}=0.707$ and 0.746 and $\mathrm{p}=0.444$ and 0.854 respectively).

Maternal heart failure was the most common cardiovascular complication encountered, occurring in seven women, all required diuretics. The majority (six out of seven) were asymptomatic pre-pregnancy with only one of these requiring early delivery (33 weeks by caesarean section) because of worsening symptoms, three required inpatient care. One patient, NYHA Class II at the onset of 
pregnancy deteriorated to NYHA Class IV by 19 weeks gestation and required inpatient care until delivery.

Six pregnancies were complicated by arrhythmia. Arrhythmia during pregnancy was more common if there was a prior history (five out of six with prior history vs one out of 48 with no prior history, $\mathrm{p}<0.0001$ by Fisher's exact test). Only one of the five women who had a documented prior arrhythmia had a previous ablation. One patient had atrial fibrillation at 15 weeks and following delivery; both episodes were treated with cardioversion. One patient was treated with amiodarone for supraventricular tachycardia prior to delivery. Ten women had at least one documented episode of hypoxemia that developed for the first time during pregnancy.

Postnatally one woman developed multiple pulmonary emboli early in the postpartum period, associated with poor compliance with LMWH. There were no cases of protein losing enteropathy prior to or during pregnancy.

\section{Obstetric complications}

Median gestation at live birth was 33.5 weeks (range 25-40 weeks, IQR 30-37 weeks). Delivery occurred before 37 weeks in 38 of 53 (71.7\%) cases. In 14 of $38(30.2 \%)$ cases this was due to spontaneous preterm labour. 13 of 38 (24.5\%) babies were delivered preterm for fetal reasons and 11 of 38 (20.8\%) for combined fetal and maternal indications. 12 out of 53 births (22.6\%) were spontaneous vaginal delivery, 10 women (18.9\%) had an assisted vaginal delivery and 31 (58.5\%) were delivered by caesarean section (12 elective, 19 emergency).

Median blood loss in the 53 livebirths was 750mls (range 160-3000mls, IQR 500-1200mls). It exceeded 500ml (classified as a PPH) in 23 (43.4\%) cases. Blood loss was lowest with spontaneous vaginal delivery $(n=13$, median $600 \mathrm{mls}$, range $200-2500 \mathrm{mls}$, IQR $300-1450 \mathrm{mls})$ and elective caesarean section ( $\mathrm{n}=12$, median $600 \mathrm{mls}$ range $300-1000 \mathrm{mls}$, IQR 500-788mls). It was higher following assisted vaginal delivery $(\mathrm{n}=10$, median $875 \mathrm{mls}$ range $200-2500 \mathrm{mls}$, IQR 525$1975 \mathrm{mls})$ and emergency caesarean section ( $\mathrm{n}=19$, median $800 \mathrm{mls}$ range 1603000mls, IQR 600-1800mls). Major obstetric hemorrhage (blood loss $>2000 \mathrm{mls}$ ) 
occurred in 7 women. There was no relationship between the use of anticoagulation regimes and postpartum blood loss.

Two pregnancies were complicated by pre-eclampsia, with cases of PIH or HELLP.

\section{Neonatal Complications}

$39(72.2 \%)$ babies were born before 37 weeks, 11 of 39 (28.2\%) following prelabour rupture of membranes. 32 of 39 (82.1\%) required admission to a neonatal unit secondary to immaturity. 30 out of $54(55.6 \%)$ babies were small for gestational age $\left(<10\right.$ th centile) with 19 out of $53(35.2 \%)<5^{\text {th }}$ centile. The median birth weight centile was nine ( $\mathrm{n}=54$, range 1-74, IQR 4-25). There was one stillbirth at 30 weeks gestation (thought secondary to severe fetal growth restriction). There were four neonatal deaths of babies born at: 26 weeks (birthweight centile 4), 27 weeks (birthweight centile 15), 31 weeks (birthweight centile 2) and 31 weeks (birthweight centile 16). All had respiratory distress syndrome and two had necrotizing enterocolitis. No babies had CHD.

\section{Cardiac complications at follow up.}

Four women defaulted from follow-up after pregnancy. Follow-up data were available for 39 pregnancies in 33 women (median follow-up was 6 years, range 4 months to 10.4 years,). There was no observed decline in ventricular function or atrio-ventricular valve regurgitation, and no maternal deaths at last follow up. Two women required cardioversion after pregnancy for recurrent atrial arrhythmias (at four and six years respectively) with one women undergoing revision of her Fontan with a modified right atrial MAZE.

\section{Discussion}

In this cohort of women with a Fontan circulation, the majority of those who conceived and went on to have a livebirth did not encounter serious maternal cardiac complications. This is likely to be because women who fail to conceive, or who conceived and subsequently miscarried, have a more advanced physiology with lower oxygen saturations. It is, also, almost certainly indicative of the 
successful provision of a specialized multi-disciplinary approach to the management of this complex group.

Although the majority of women in our cohort were NYHA class I or had a CARPREG score of 0 or 1 (86\%) pre-pregnancy, we observed a high rate of miscarriage, obstetric and neonatal complications. The background rate of miscarriage in women with a clinically apparent pregnancy, but without heart disease is $20 \%$ (13). The rate of miscarriage in our study was $56 \%$. This is double the rate of miscarriage in women with most other forms of congenital heart disease (14) and similar to the high rates of miscarriage ( $>40 \%)$ reported in large series of women who had cyanotic heart disease (15). Presbitero et al demonstrated that the high rate of miscarriage in women with cyanotic heart disease relates to lower maternal oxygen saturations and higher baseline maternal haemoglobin concentrations (15). It may be that the high rate of pregnancy complications is related to an adverse effect of the Fontan circulation on the venous circulation in the pelvis. Interestingly, within our cohort $24 \%$ (13 of 54) of women had a fenestrated Fontan of which the majority had saturations of $<92 \%$. Whilst cyanosis has a demonstrated association with miscarriage, which we also found in this cohort, the impact of a fenestration in the Fontan circulation is less clear and from our results does not appear to adversely affect the likelihood of achieving a viable pregnancy. The relationship between cyanosis and fenestration is complex as there are other causes for cyanosis in the Fontan circulation such as collateral formation.

Reassuringly most women had PCC although the nature of this will vary between centres. This is higher than a recent study that examined rates of PCC in women with heart disease (16) . Whilst maternal events were low in our cohort, consideration should be given at the time of PCC as to whether elective ablation in the context of prior arrhythmia or, more controversially, strategies to optimise saturations should be considered for women with Fontan circulations embarking on pregnancy.

Obstetric events were common, with $72 \%$ of women delivering pre-term with $44 \%$ of these births due to spontaneous preterm labour. Spontaneous preterm labour is commoner in women with heart disease, but this is poorly 
understood $(17,18)$. Median birth weight centile was nine suggesting that most babies were growth restricted as well as being born preterm. Small for gestational age babies are commoner in women with CHD, but the degree of growth restriction in our series is greater than reported in other forms of heart disease(19). Kapman et al linked cardiac disease with abnormal placentation, demonstrating abnormal uteroplacental Doppler flow studies in women with pre-pregnancy cardiac dysfunction $(20,21)$. Serial growth scans are indicated from 24 weeks gestation due to the significantly increased risk of SGA.

PPH was more common than expected with an overall rate of $43 \%$. Previously, we have shown that women with a Fontan circulation have a greater risk of PPH than women with other cardiac lesions (22). In 13\% of deliveries (seven of 54) women had a major obstetric haemorrhage (blood loss $>2000 \mathrm{mls}$ ). Blood loss at delivery was lowest with spontaneous vaginal delivery, which is consistent with data from the Registry on Pregnancy and Cardiac Disease (ROPAC) (23). However, blood loss was greatest in women who underwent an assisted vaginal delivery, largely as a result of perineal trauma (24). Since many women with a Fontan circulation maybe advised to have a shortened second stage (25), the rate of assisted vaginal delivery may contribute to the higher rate of PPH. Interestingly, blood loss did not correlate with the anticoagulation regimen.

The preferred strategy for anticoagulation is controversial; the concern of thromboembolic complications secondary to the pro-thrombotic combination of the Fontan circulation and pregnancy. Currently there is no consensus on which anticoagulation regime to give (26). Using aspirin and warfarin outside of pregnancy reduces the incidence of thromboembolic events although it remains unclear which strategy is optimal (27). Since both a Fontan circulation and pregnancy are pro-thrombotic conditions, it would seem reasonable to use anticoagulants to reduce this risk, however the evidence is consensus opinion. Finally, there remains significant uncertainty and unease with regards to the long-term impact of pregnancy upon the Fontan circulation.

\section{Limitations}


Our study was retrospective so is susceptible to missing data. Under-reporting of miscarriage and termination of pregnancy is likely. Our cohort is limited to women who were NYHA class I or II, with no women with protein losing enteropathy. This may be due to these women being counseled against pregnancy, or because they fail to conceive. Our study was multicenter and there was no standardized practice for managing this group of women in pregnancy. We are therefore unable to draw conclusions with regards to the long-term impact of pregnancy upon women with the Fontan circulations. We have been able to comment on associations found to be significant but there may be some relevant associations that were not apparent in our study simply by chance because of the relatively small numbers of patients on whom we had data.

\section{Conclusion}

Women who conceive with a Fontan circulation and particularly those who have low oxygen saturations have high rates of miscarriage. Those that do not miscarry are more likely to encounter obstetric and neonatal complications; in particular higher rates of preterm delivery, fetal growth restriction and PPH. Adverse cardiac events are relatively uncommon when women are managed in a specialized multi-disciplinary setting.

The Corresponding Author has the right to grant on behalf of all authors and does grant on behalf of all authors, an exclusive licence (or non exclusive for government employees) on a worldwide basis to the BMJ Publishing Group Ltd and its Licensees to permit this article (if accepted) to be published in HEART editions and any other BMJPGL products to exploit all subsidiary rights"

\section{Competing interest: None}

Funding: None 
Contribution to authorship: MC conceived the idea for the manuscript and contacted all other authors who assisted in providing data. PJS and MC analysed the data. MC, PJS and MJ wrote the first draft and this was edited and approved by all authors. 


\section{References}

1. Coats L, O'Connor S, Wren C, O'Sullivan J. The single-ventricle patient population: a current and future concern a population-based study in the North of England. Heart. 2014;100(17):1348-53.

2. Gewillig M, Brown SC. The Fontan circulation after 45 years: update in physiology. Heart. 2016;102(14):1081-6.

3. Drenthen W, Pieper PG, Roos-Hesselink JW, van Lottum WA, Voors AA, Mulder BJM, et al. Pregnancy and delivery in women after Fontan palliation. Heart. 2006;92(9):1290-4.

4. Cauldwell M, Von Klemperer K, Uebing A, Swan L, Steer PJ, Babu-Narayan SV, et al. A cohort study of women with a Fontan circulation undergoing preconception counselling. Heart (British Cardiac Society). 2016;102(7):534-40. 5. Ohuchi H, Yasuda K, Miyazaki A, Ono S, Hayama Y, Negishi J, et al. Prevalence and predictors of haemostatic complications in 412 Fontan patients: their relation to anticoagulation and haemodynamics. European journal of cardio-thoracic surgery : official journal of the European Association for Cardiothoracic Surgery. 2015;47(3):511-9.

6. Gouton M, Nizard J, Patel M, Sassolas F, Jimenez M, Radojevic J, et al. Maternal and fetal outcomes of pregnancy with Fontan circulation: A multicentric observational study. Int J Cardiol. 2015;187:84-9.

7. Zentner D, Kotevski A, King I, Grigg L, d'Udekem Y. Fertility and pregnancy in the Fontan population. International Journal of Cardiology. 2016;208:97-101.

8. Siu SC, Sermer M, Colman JM, Alvarez AN, Mercier LA, Morton BC, et al. Prospective multicenter study of pregnancy outcomes in women with heart disease. Circulation. 2001;104(5):515-21.

9. Gardosi J, Chang A, Kalyan B, Sahota D, Symonds EM. Customised antenatal growth charts. Lancet (London, England). 1992;339(8788):283-7. 10. Gardosi J, Mongelli M, Wilcox M, Chang A. An adjustable fetal weight standard. Ultrasound in obstetrics \& gynecology : the official journal of the International Society of Ultrasound in Obstetrics and Gynecology. 1995;6(3):168-74.

11. Gynaecologists RCoOa. Reducing the Risk of

Venous Thromboembolism during Pregnancy and the Puerperium. 2015.

12. Broberg CS, Jayaweera AR, Diller GP, Prasad SK, Thein SL, Bax BE, et al. Seeking optimal relation between oxygen saturation and hemoglobin concentration in adults with cyanosis from congenital heart disease. The American journal of cardiology. 2011;107(4):595-9.

13. Nybo Andersen AM, Wohlfahrt J, Christens P, Olsen J, Melbye M. Maternal age and fetal loss: population based register linkage study. BMJ (Clinical research ed). 2000;320(7251):1708-12.

14. Khairy P, Ouyang DW, Fernandes SM, Lee-Parritz A, Economy KE, Landzberg MJ. Pregnancy outcomes in women with congenital heart disease. Circulation. 2006;113(4):517-24. 
15. Presbitero P, Somerville J, Stone S, Aruta E, Spiegelhalter D, Rabajoli F. Pregnancy in cyanotic congenital heart disease. Outcome of mother and fetus. Circulation. 1994;89(6):2673-6.

16. Cauldwell M, Ghonim,S. Preconception couselling, predicting risk and outcomes in women with mwho 3 and 4 heart disease International Journal of Cardiology. 2017.

17. Siu SC, Colman JM, Sorensen S, Smallhorn JF, Farine D, Amankwah KS, et al. Adverse neonatal and cardiac outcomes are more common in pregnant women with cardiac disease. Circulation. 2002;105(18):2179-84.

18. Makino Y, Matsuda Y, Mitani M, Shinohara T, Matsui H. Risk factors associated with preterm delivery in women with cardiac disease. Journal of cardiology. 2012;59(3):291-8.

19. Gelson E, Curry R, Gatzoulis MA, Swan L, Lupton M, Steer P, et al. Effect of maternal heart disease on fetal growth. Obstetrics and gynecology. 2011;117(4):886-91.

20. Kampman MA, Bilardo CM, Mulder BJ, Aarnoudse JG, Ris-Stalpers C, van Veldhuisen DJ, et al. Maternal cardiac function, uteroplacental Doppler flow parameters and pregnancy outcome: a systematic review. Ultrasound in obstetrics \& gynecology : the official journal of the International Society of Ultrasound in Obstetrics and Gynecology. 2015;46(1):21-8.

21. Wald RM, Silversides CK, Kingdom J, Toi A, Lau CS, Mason J, et al. Maternal Cardiac Output and Fetal Doppler Predict Adverse Neonatal Outcomes in Pregnant Women With Heart Disease. Journal of the American Heart Association. 2015;4(11).

22. Cauldwell M, Von Klemperer K, Uebing A, Swan L, Steer PJ, Gatzoulis M, et al. Why is post-partum haemorrhage more common in women with congenital heart disease? Int J Cardiol. 2016;218:285-90.

23. Ruys TP, Roos-Hesselink JW, Pijuan-Domenech A, Vasario E, Gaisin IR, Iung $\mathrm{B}$, et al. Is a planned caesarean section in women with cardiac disease beneficial? Heart. 2015;101(7):530-6.

24. Gurol-Urganci I, Cromwell DA, Edozien LC, Mahmood TA, Adams EJ, Richmond DH, et al. Third- and fourth-degree perineal tears among primiparous women in England between 2000 and 2012: time trends and risk factors. Bjog. 2013;120(12):1516-25.

25. Regitz-Zagrosek V, Blomstrom Lundqvist C, Borghi C, Cifkova R, Ferreira R, Foidart JM, et al. ESC Guidelines on the management of cardiovascular diseases during pregnancy: the Task Force on the Management of Cardiovascular Diseases during Pregnancy of the European Society of Cardiology (ESC). Eur Heart J. 2011;32(24):3147-97.

26. Pundi KN, Pundi K, Johnson JN, Dearani JA, Bonnichsen CR, Phillips SD, et al. Contraception Practices and Pregnancy Outcome in Patients after Fontan Operation. Congenit Heart Dis. 2016;11(1):63-70.

27. Potter BJ, Leong-Sit P, Fernandes SM, Feifer A, Mayer JE, Jr., Triedman JK, et al. Effect of aspirin and warfarin therapy on thromboembolic events in patients with univentricular hearts and Fontan palliation. Int J Cardiol. 2013;168(4):3940-3. 